\title{
On the Mechanical Properties and Thermal Stability of a Recently Synthesized Monolayer Amorphous Carbon
}

\author{
Levi C. Felix ${ }^{1}$, Raphael M. Tromer ${ }^{2}$, Pedro A. S. Autreto ${ }^{3}$, Luiz A. Ribeiro Juniorr ${ }^{4}$, and \\ Douglas S. Galvao ${ }^{12} *$ \\ ${ }^{1}$ Applied Physics Department, State University of Campinas, Campinas/SP, 13083-970, \\ Brazil \\ ${ }^{2}$ Center for Computational Engineering \& Sciences - CCES, State University of Campinas, \\ Campinas/SP, 13083-970, Brazil. \\ ${ }^{3}$ Center of Natural and Human Sciences, Federal University of ABC, 70919-970, Santo \\ André, SP, Brazil. \\ ${ }^{4}$ Institute of Physics, University of Brasília, 70919-970, Brasília, Brazil. \\ *corresponding author: galvao@ifi.unicamp.br
}

This electronic supplementary information presents additional data concerning statistical analysis for the linear fitting performed on the data presented in Figure 4 in the main manuscript and energy components as a function of time.

\section{Temperature ramp}

Due to the presence of temperature fluctuations in the molecular dynamics simulations, the correspondence between time and temperature is obtained from a linear fitting in order to express energy values as a function of temperature, as shown in Figure 4 of the main manuscript.

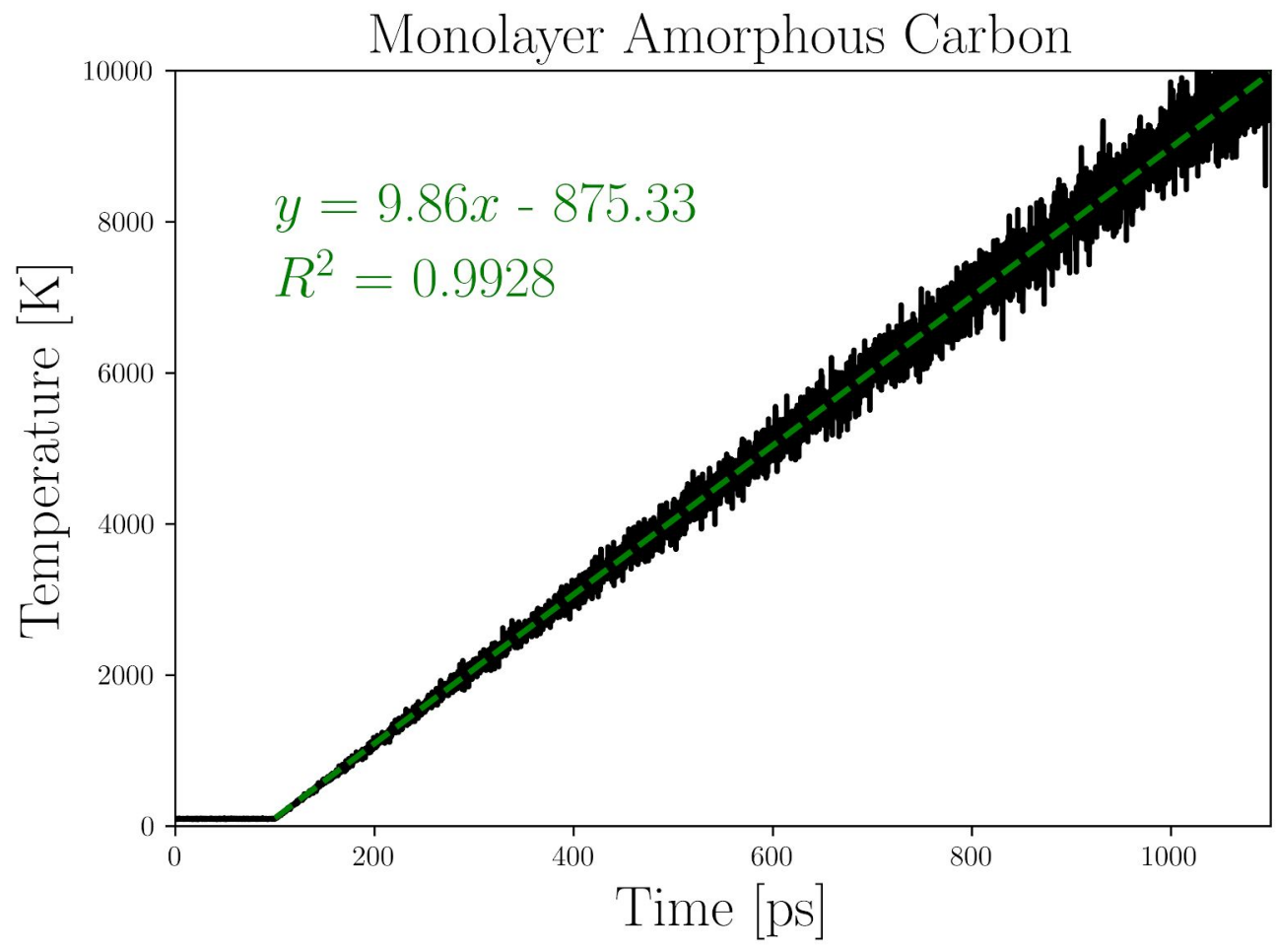

Figure S1. Linear fitting of temperature as a function of time. 


\section{Energy contributions during the heating process}

Figure S2 shows that as the temperature (consequently, the kinetic energy) increases. As the total energy also increases, the potential energy decreases to a nonzero value due to the presence of dimers, trimers and short atomic carbon chains shown in Figure 5 of the main manuscript. The potential energy is given by the formula

$$
V=E_{R E B O}+E_{L J}+E_{\text {TORSION }},
$$

where $E_{R E B O}, E_{L J}$, and $E_{T O R S I O N}$ are the bond order, Lennard-Jones, and Torsional terms, respectively.

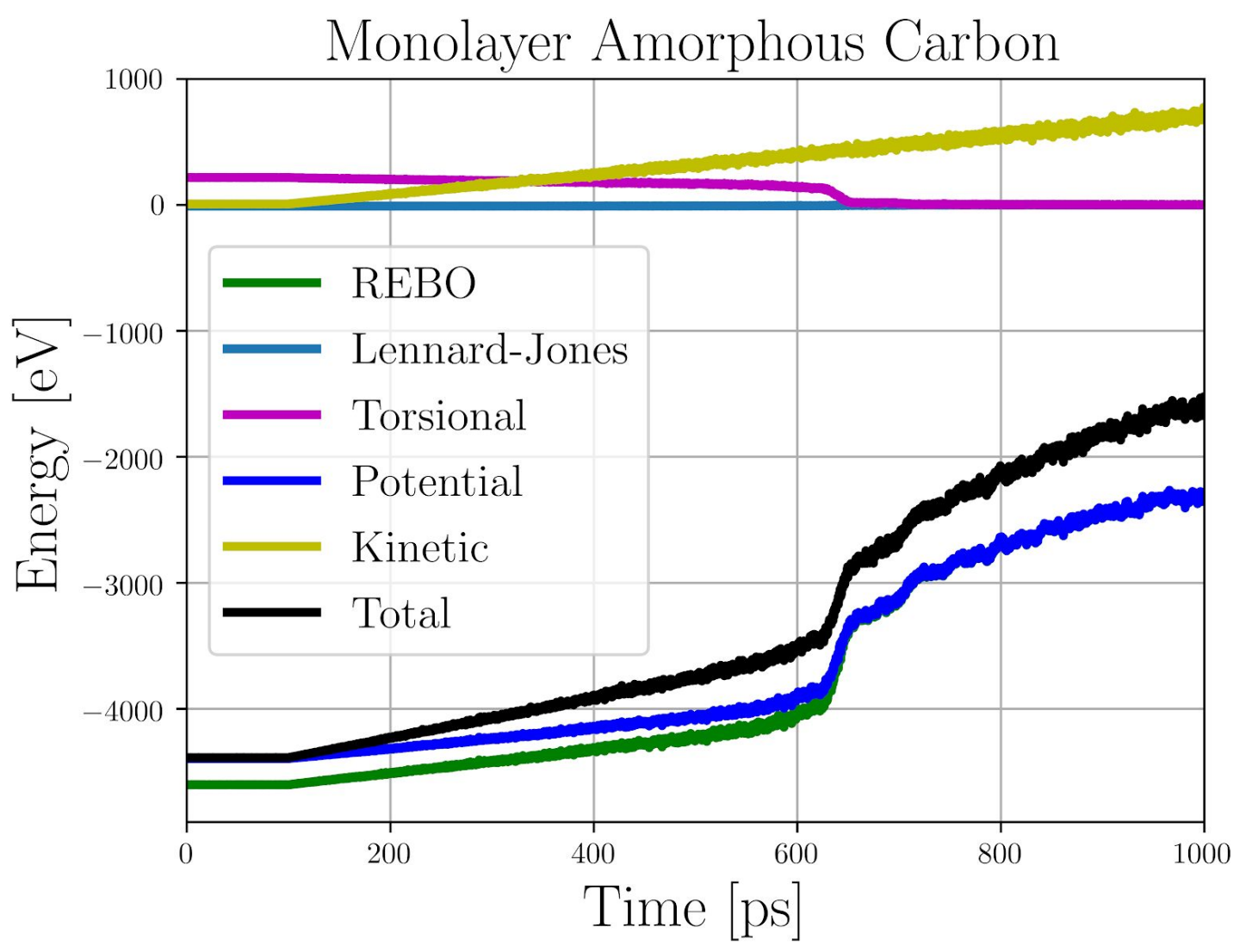

Figure S2. Energy as a function of time to highlight all different contributions to the total energy. 